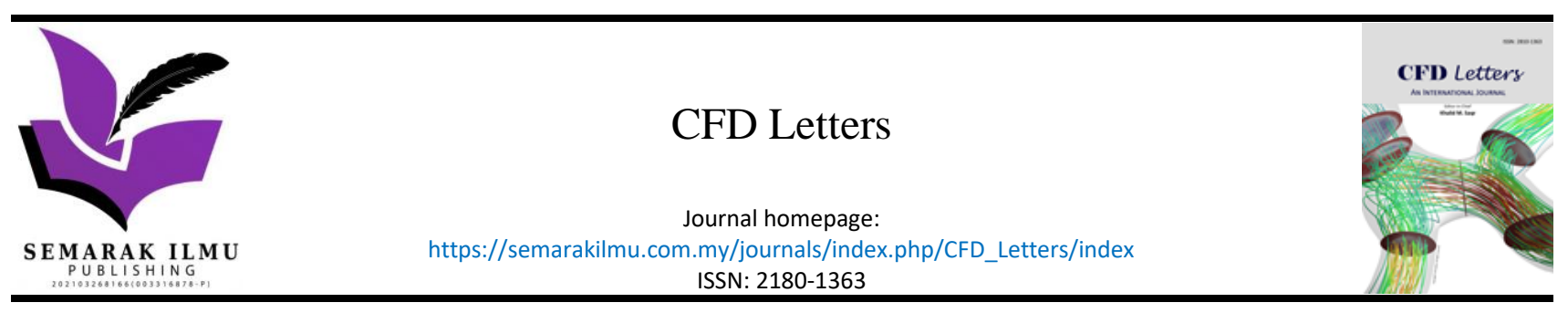

\title{
Porosity Effect of the Silver Catalyst in Hydrogen Peroxide Monopropellant Thruster
}

\author{
Muhammad Shahrul Nizam Shahrin ${ }^{1}$, Norazila Othman ${ }^{1, *}$, Nik Ahmad Ridhwan Nik Mohd ${ }^{1}$, Mastura \\ Ab Wahid ${ }^{1}$, Mohd Zarhamdy Md. Zain ${ }^{1}$
}

1 School of Mechanical Engineering, Faculty of Engineering, Universiti Teknologi Malaysia, 81310 Skudai, Johor, Malaysia

ARTICLE INFO

\section{Article history:}

Received 13 July 2021

Received in revised form 31 October 2021

Accepted 1 November 2021

Available online 14 December 2021

\section{Keywords:}

Hydrogen peroxide monopropellant thruster; silver screen catalyst; pressure drop across catalyst bed; porosity of catalyst bed

\section{ABSTRACT}

\begin{abstract}
In monopropellant system, hydrogen peroxide is used with catalyst to create an exothermic reaction. Catalyst made of silver among the popular choice for this application. Since the catalyst used is in porous state, the effect of its porosity in the hydrogen peroxide monopropellant thruster performances is yet unknown. The porosity changes depending on factors including catalyst pact compaction pressure, bed dimension, and type of catalyst used. As researches on this topic is relatively small, the optimum porosity value is usually left out. The performance of the thruster indicated by the pressure drop across the catalyst bed. Porosity of the catalyst bed adds additional momentum sink to the momentum equation that contributes to the pressure gradient which lead to pressure loss inside thruster. The effect of porosity influences the performance and precision of the thruster. Study of the pressure drop by the catalyst bed requires a lengthy period and expensive experiments, however, numerical simulation by mean of Computational Fluid Dynamics (CFD) can be an alternative. In this paper, $90 \mathrm{wt} \%$ hydrogen peroxide solution with silver catalyst is studied in order to investigate the influence of porosity to the performances of the thruster, and to find the optimum porosity of the thruster. Species transport model is applied in the single-phase reaction simulation using the EDM for turbulencechemistry interaction. Through this study, the effect of porosity towards the thruster performances represented in term of pressure drop, exit velocity, bed temperature, and thrust, and porosity of 0.4 found to be as an optimal value.
\end{abstract}

\section{Introduction}

Propulsion technology in space science usually divided into three main categories which are escape propulsion, in-space propulsion, and deep space propulsion [1]. Propulsion technologies made up of several propulsion system such as chemical powered (in form of solid, liquid, hybrid, and green propellant), non-chemical propulsion such as plasma (electric or ionic propulsion), advanced propulsion (thermo-nuclear based propulsion, laser, and fusion), and propellant-less propulsion (solar sail) [2]. Chemical propulsion is the earliest form of propulsion system and it is typically made up of solid or liquid type propellant. Liquid propellant usually either in a form of monopropellant or

\footnotetext{
*Corresponding author.
}

E-mail address: norazila@mail.fkm.utm.my (Norazila Othman) 
bi-propellant depending on how it was made while hybrid propulsion made up of combination of both solid and liquid propellants.

Liquid propulsion is a system that utilized liquid chemical in producing thrust via combustion process. The only different between mono and bi-propellant is that the later used a combination of propellant and oxidizer while monopropellant uses single chemical that does not required a separate oxidizer. Monopropellant thruster is ideal for small satellite or can be used in CubeSats and to be used on various applications such as orbital insertion, orbit raising, station keeping, spin control, and satellite decommissioning [3-5]. It can also be used on the launch vehicles as an igniter or as an attitude controller [5].

Solid fuel has a good record and performance but it has major disadvantages that are its thrust output cannot be controlled, and it cannot be switched off once it had burnt, which make it unsuitable to be used as a control thruster [1]. On the other hand, bi-propellant even though it behaves much better in term of output control and can be turn on and off but since it requires additional separate oxidizers creating additional cost in term of weight and space which make it too complicated for similar applications. For this reason, monopropellant usually used in control thruster application, which motivates us to invest in the study of monopropellant thruster.

Propulsion and control system for satellites and spacecraft dominated by the hydrazine-based propellants [6]. Monomethylhydrazine $(\mathrm{MMH})$, unsymmetrical dimethylhydrazine (UDMH), and A 50 (hydrazine and UDMH at 50/50 mixture) are the derivatives of hydrazine, and oxidizers such as NTO, MON, RFNA and WFNA are considered very toxic and carcinogenic [7]. These propellants demand an ecological challenge, and utilization of these materials create a burden to the health and safety for the working personnel [8]. Future space activities and applications are broadening at greater rate, hence, more environmentally space propulsion system will be significantly increasingly desired. Over the last few years, low toxicity and less carcinogenic liquid propellants is an increasingly become a discussed subject as a potential candidate to replace the hydrazine-based propellants $[1,8,9]$.

Numbers of propellants investigated found to carry the material and chemical properties that capable to be a good green propellant [10]. Green propellants usually can be exploited as a propellant or oxidizer in both mono and/or bipropellant system. Green propellants make a significant reduction in toxicity, having a reliable potential in term of performance, easier to handle and store, and in some cases, it is cheaper [10]. Few types of propellants have been tested including ammonium dinitramide (AND), ammonium nitrate (AN), hydrogen peroxide, ethanol, heptane, kerosene (Jet-A1), dLimonene, octane, turpentine and solid fuel [10]. According to Gohardani et al., [11] hydrogen peroxide, nitrous oxide fuel blends (NOFB) and ionic liquids could be a substitute for monopropellant hydrazine.

Hydrogen peroxide is a versatile material that can be used as a monopropellant or oxidizer in bipropellant system. It is thermodynamically unstable, very reactive, decomposes slowly even in its most stabilized form, and possess a theoretically lower specific impulse than that of hydrazine [11]. However, hydrogen peroxide blended ethanol has a potential to be high-performance propellant in a monopropellant which possess equal or greater performance than hydrazine [9]. Rocket grade hydrogen peroxide or high-test peroxide (HTP) can be a fuel igniter in bipropellant system in addition to the oxidizer [12]. Sobczak et al., [12] listed main advantages of the hydrogen peroxide which are nontoxic, easy to handle, performance on par with the MMH/MON propellants, ability to selfignition, and unlimited restart-ability.

Research on hydrogen peroxide monopropellant thruster generally show that environment friendlier propulsion system is possible. In addition to that, this study is in accordance with the National Policy on Environment that requires commitment and proactive participation from 
researchers and institutes of higher education in achieving the policy objectives. Furthermore, the benefits of the study not only limited to the environment policies but most importantly to the development of research framework (research and development of the monopropellant thruster) in respond to the Malaysian Aerospace Industry Blueprint 2030 under sub-sector Engineering and Design [13].

A research by Rhodes and Ronney [3] reveals that hydrogen peroxide is good for small satellite which could provide millinewtons of thrust at specific impulse $\left(I_{s p}\right)$ around 200s. The use of hydrogen peroxide vapor in small thruster unit may contribute to the designed thrust from $0.5 \mathrm{mN}$ to $8 \mathrm{mN}$ by varying the operating temperature [3]. Huh et al., [14] stated that, propellants such as HAN and ADN are good alternatives for monopropellant thruster but due to their high viscosity, contributes to a high feeding pressure loss make it less suitable for small thruster. Besides, they required preheating and as oppose to hydrogen peroxide make them more suitable for microthruster [14]. A similar study has been done by Kuan et al., [15] their hydrogen peroxide monopropellant microthruster able to produce $182 \mathrm{mN}$ of thrust with 101 second specific impulse at sea level by utilizing $92 \%$ HTP and silver catalyst. Hence, hydrogen peroxide shows a reliable material as a monopropellant with a good performance without sacrificing the environment.

HTP (roughly more than 85 wt.\% concentration) has many usages in aerospace application especially as a green propellant option $[8,16]$. It has many advantages over other type of conventional hydrazine-based propellant in terms of storing, workability and environment. Despite the benefits, it faces challenges in proving its performance because it seemed inferior compared to traditional propellant $[17,18]$. However, with recent studies and developments by scientist and engineers, significant improvement in terms of performances and other attributes can be seen and hydrogen peroxide is expected to play a bigger role in aerospace activities in near future.

Monopropellant thruster uses single type of fuel or fuel-blend to generate thrust force. Many researches and experiments have been done using the hydrogen peroxide at various concentrations with different types of catalysts. Few works can be observed by utilizing silver as catalyst such as in the works of Shahrin et al., [19] and Othman et al., [20] in a development of 50N class monopropellant thruster using 90\% concentration hydrogen peroxide, and work from Kuan et al., [15] where $100 \mathrm{mN}$ microthruster is developed using $0.7 \mathrm{~g}$ of silver flakes in the catalyst bed, producing a result of $90 \% \mathrm{C}^{*}$ efficiency and 101s of $I_{s p}$. Another work to be noted is by Cervone et al., [21] which tested their $5 \mathrm{~N}$ and $25 \mathrm{~N}$ monopropellant thrusters by using $87.5 \%$ concentration hydrogen peroxide with pure silver gauge and pallets as catalyst, coated with manganese oxide and platinum. Silver catalyst performance characteristics i.e., exhaust velocity and pressure drop; and its own reaction with hydrogen peroxide has also been studied and reported by Lee and Lee [22]. The development of the hydrogen peroxide monopropellant thruster can be observed by the work of Amri et al., [23], and Haq et al., [24] where both of them developed the $1 \mathrm{~N}$ class thruster by using HTP and silver catalyst.

Roughly, silver (usually in form of silver screen) is seeming as a popular choice as the catalyst. This is simply because it is widely available in the market and the price can be said quite competitive in addition to its performance and reliability. Therefore, the conveniences of using this type of material shall be discussed in this article.

\subsection{Thruster Working Mechanism}

Monopropellant thruster consist of few basic components as shown in Figure 1, which include pressurize gas tank, propellant tank, combustion chamber, nozzle, valves and piping. Typically, there are two types of feeding system available which are pressure fed or pump fed system where both 
pressurize gas tank and pump have the same purpose, which is to deliver the propellant injection into the combustion chamber [24]. Usually for pressure fed system, inert gas like nitrogen is used.

By referring to Figure 1 and Figure 2, the flow mechanism inside the thruster started when propellant receives pressure from the pressurized gas, it is then channel into the combustion chamber via propellant inlet that is controlled by the valves in order to get the right injection pressure. Similar concept is used in the pump fed system where propellant is pumped into the chamber. Through injector, propellant will be sprayed onto the catalyst bed. The type of injection scheme is depending on the type of injection orifice used as well as the inlet pressure that contribute to the depth and size of the spray cone. Injected propellant is then entering the catalyst bed and undergoes chemical reaction with the catalyst material; which then the products of the said reaction is released through exit nozzle in form of thrust force. The internal structures of the combustion chamber are varied from one design to another depending on the purpose of the thruster itself.

From Figure 2, the thruster chamber depicted consists of propellant inlet that acts as a channel for propellant to enter the chamber, injector which acts as a 'door' that control the behaviour of the sprayed propellants. Distribution plates in front and aft of the catalyst bed are to promote uniform flow of the propellant downstream the catalyst bed (and exiting into the nozzle section), and they also act as barriers to guard the shattered catalyst that maybe undergo some sort of disorientation through attrition and sintering phenomenon that take place during the decomposition process with hydrogen peroxide. Due to the weakening of the structural integrity of catalyst, aft distribution plate prevents catalyst from exiting off the chamber. The catalyst bed is designed to hold the catalyst materials, it is also a place where all the reaction happens. The nozzle also known as de Laval nozzle is a component where the products of the reactions going to be delivered to produce thrust force. Nozzle consist of three main division which are inlet, throat, and exit nozzle. The geometry and sizing of the nozzle varies from one to another depending on the use and mission of the particular thruster engine.

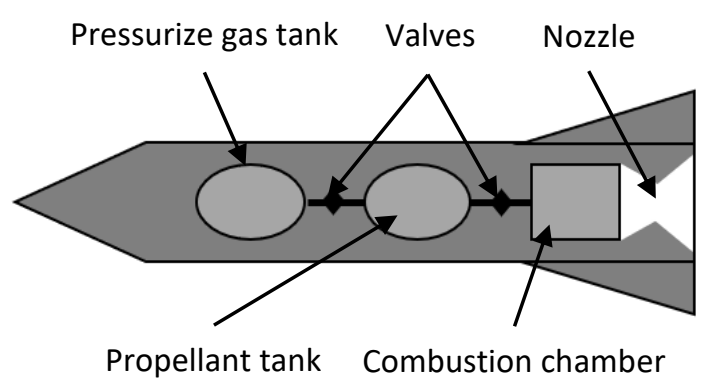

Fig. 1. Hydrogen peroxide monopropellant thruster

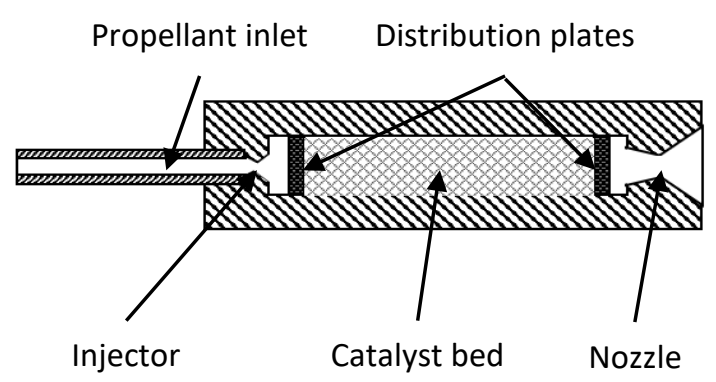

Fig. 2. Thruster combustion chamber adapted from Shahrin et al., [19]

\subsection{Decomposition of Hydrogen Peroxide}

In hydrogen peroxide monopropellant system, simple reaction of hydrogen peroxide solution is exploited to get the thrusting force. It will undergo decomposition reaction when reacts with catalyst or heat where hydrogen peroxide will dissociate into oxygen gas and water steam. This behaviour is depicted in the Eq. (1) where, $Q$ is the unit of exothermic energy released by the reaction.

$2 \mathrm{H}_{2} \mathrm{O}_{2} \stackrel{\text { Catalyst }}{\rightarrow} 2 \mathrm{H}_{2} \mathrm{O}+\mathrm{O}_{2}+Q$ 
Hydrogen peroxide $\left(\mathrm{H}_{2} \mathrm{O}_{2}\right)$ is a compound with two oxygen atoms connected via single bond and it is the simplest form of peroxides. In nature, the peroxide bond is quite unstable and tend to decompose. So, it is kept with additive in form of stabilizers such as phenol, sodium stannate, tetrasodium pyrophosphate, and acetanilide. The decomposition process of hydrogen peroxide which also known as a disproportionation reaction is described in Figure 3, showing that the oxygen atom in hydrogen peroxide $\left(\mathrm{H}_{2} \mathrm{O}_{2}\right)$ is at -1 oxidation state, and then oxidized to form oxygen molecule $\left(\mathrm{O}_{2}\right)$ with oxidation state of 0 magnitude as well as at the same time reduce to form water $\left(\mathrm{H}_{2} \mathrm{O}\right)$ with oxidation state of -2 [25].

Hydrogen peroxide are quite easy to react (decompose) if disclosed to impurities or catalysts materials like metallic surfaces or yeast. The rate of reaction can be influenced by so many factors such as temperature, pressure, concentration, type of catalyst, and area of active catalytic surface of the catalyst, as well as an exposure to direct sunlight, and presence of inhibitors [25]. However, the reaction rate is considered slow in mild temperature without the presence of the catalytic materials, hence it can be stored without losing its properties for long time. The speed of reaction will rise with increasing temperature; this reaction called thermal decomposition reaction. Similarly, the presence of catalytic material will enhance it rate of reaction.

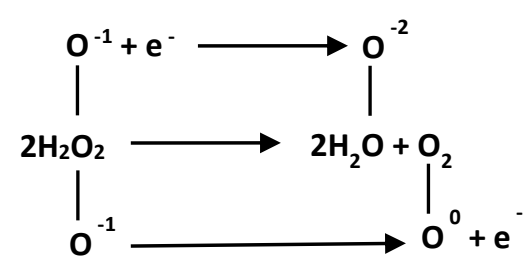

Fig. 3. Reaction of disproportionation of hydrogen peroxide adapted from Pędziwiatr et al., [25]

The reaction can produce huge amount of heat $(-2884.5 \mathrm{~kJ} / \mathrm{kg}$ for pure compound), which further risen the reaction rate and turn it into self-sustaining after the phase of catalytic initiation [25]. The reaction mechanism is relying on the type of catalyst used. However, conclusive reactions mechanism is yet to be determined since there are so many postulations circulated e.g., radical mechanism, and complex mechanism, to name a few [26]. Besides, thermal decomposition reaction is also a very complex process which led to the idea that the final reaction mechanism is a mix and combination of multitype of mechanisms, where thermal decomposition is included to the reaction with catalyst and impurities [26]. In addition, the decomposition process of hydrogen peroxide also can be done in both phases; liquid form and vapor form. According to Božic et al., [26] they stated that the catalytic decomposition of hydrogen peroxide exhibits a zero order or first order reaction based on the type of catalyst used.

\subsection{Silver Catalyst}

A catalyst is a material that affects, enhances, or expedites reaction by means of increasing the rate of reaction without being consumed in the process. Generally, it accelerates a reaction process by lowering the activation energy or changing the reaction mechanism. Activation energy is the least amount of energy needed to transform a stable molecule to becomes a reactive molecule. There are many types and forms of catalyst. It can be categorized into two forms based on phase homogeneity, i.e., homogeneous form and heterogeneous form. Homogeneous catalyst is a catalyst that have same 
phase with the liquid hydrogen peroxide while heterogeneous catalyst is at different phase usually in solid phase.

Hydrogen peroxide can easily undergo decomposition reaction with presence of many types of catalytic material. For examples, iodide, iron ions, manganese, $\mathrm{Al}_{2} \mathrm{O}_{3}, \mathrm{MnO}_{2}$, silver, gold, iron, platinum, catalase (enzyme catalyst), and many more. Several papers found discussing other type of catalyst for hydrogen peroxide decomposition such as by Essa et al., [8] and Widdis et al., [27] that used manganese oxides, and ruthenium oxides nanorods respectively. According to Widdis et al., [27] their experiment on the ruthenium oxides showed that the decomposition took place on the catalytic surfaces, but the magnitude of reactivity recorded is not as anticipated for the decomposition of $85 \%$ hydrogen peroxide. According to Gagne [28], Iron compounds were found to be among the most vigorous hydrogen peroxide decomposition out of all the catalysts investigated while iron salts is one of the highest performing catalysts especially in the hydrogen peroxide propellant. While according to Khaji et al., [29] the catalytic performance for both silver and platinum are almost similar even though platinum is found slightly more stable in real scenario.

Utilization of silver catalyst in the hydrogen peroxide monopropellant thruster is not uncommon. It becomes a popular choice because it is widely available on the market, traditionally proven and has a strong reputation, uncomplicated safety handling and storage. Typically, silver used as catalyst is in metallic form or metal alloy. lonic form of silver is not use for this application since it is likely to induce precipitation. Usually, silver metal in form of woven mesh called screen, powder, or flakes is used. In this study, mesh type is used. Besides that, silver also used as a coating to another metal in lieu of pure metal, this is done to reduce the cost. However, the rate of reaction principally will not be affected as the reaction usually happens on the interphase layer only.

Silver catalyst has its own advantages and disadvantages but remain a popular choice for hydrogen peroxide monopropellant thruster. Pędziwiatr et al., [25] listed that there are four central advantages of the silver catalyst which are; high decomposition efficiency, uncomplicated fabrication process, compactness, and it is available in various form. Together with hydrogen peroxide it can perform with low latency for ignition delay. On the other hand, it can also help hydrogen peroxide performs restart-ability function, but unlimited restart-ability is found to be challenging to this type of catalyst due to various reasons especially regarding it life span issue.

In spite of that, it carries major difficulties due to its life span issue as it is found to be easily deteriorate when decomposed with hydrogen peroxide; hence, limiting the life of the catalyst bed itself [16]. Even though silver is said as the best catalyst for HTP in term of catalytic performance, but due to its low melting point (compared to other catalyst) it will tend to sinter during the decomposition reaction takes place [16]. This led to incomplete decomposition in cold start and oscillation in chamber pressure where it will eventually induce flow instability of the supplying system [16].

According to Kang et al., [30] there are two aspects that contribute to the deformation or deterioration of catalyst bed. Firstly, propellant injection momentum induced compressive load onto the catalyst bed which potentially make it lumped and moved back to the end of the bed. This event might motivate the latency in response time and decrease the rate of reaction due to contraction of total effective surface area of the catalyst. Secondly, Undesired distribution of catalyst can happen due to a factor of catalyst mechanical strength, i.e., catalyst attrition, and catalyst channelling. As the temperature rises, and constant flow of pressure teared the catalyst; into smaller pieces, then drove it out of the bed creating uncertain number of voids inside the catalyst bed. This can lead to the performance instabilities including exorbitant amount of pressure loss.

The silver catalyst will cause strong reaction instantaneously just after the initial injection of hydrogen peroxide which cause instantaneous heat release from hydrogen peroxide decomposition 
process, that results in sudden temperature rising in the chamber to the steady state temperature in a very short of time. The huge heat release and abrupt temperature jump will induce a sintering process in the catalyst [16]. The sintering effect will reduce the decomposition efficiency and increase the fluctuation of the chamber pressure which also could cause the supply of hydrogen peroxide erratic.

Another drawback of silver catalyst is that its melting point is quite low at only $\sim 962{ }^{\circ} \mathrm{C}(\sim 1235 \mathrm{~K})$. If compared to the temperature achieved by the decomposition process of high concentration hydrogen peroxide, it can reach temperature about $1000 \mathrm{~K}$ which are very near to the melting temperature of silver catalyst. This little offset could not provide sufficient buffer to the structural integrity of the silver as it will started to deform when it moves towards its melting temperature. This can lead to the problems mentioned before such as attrition and sintering of the silver metal as at that temperature, its structure will be too weak to hold and sustain the pressure from the flow. If that happens, it tends to accumulate and pile up at the end of catalyst bed (just before the rear distribution plate) and started to block the flow even more, resulting in increasing pressure loss across the bed. Besides, this phenomenon will also cut down the effective contact area between the silver and hydrogen peroxide which eventually led to reduction in decomposition efficiency.

Contrary to the hydrogen peroxide restart-ability characteristic, silver catalyst possesses several challenges in conducting such recurring work. Mass loss due to reaction cycles took place during decomposition reaction process. The rate of mass loss of silver is proportional to the squared area of the catalyst. This relation was explained detail by Baumgartner et al., [31] in their articles about reaction of hydrogen peroxide with silver. The concentration of silver ions in the solution stabilizes as a result of solution saturation; these ions inhibit silver degeneration. Furthermore, silver also prone to surface poisoning. However, it could be regenerated with nitric acid [25]. After regeneration, surface of metal developed which increase the number of active centres. It is essential for new silver since the surface of new silver is undeveloped and smooth which will narrow down the efficiency of decomposition [25]. So, the unlimited restart-ability possess by the hydrogen peroxide propellant might face difficulty in performing such work with heterogeneous silver metal as catalyst in particular.

Even though silver has its own pros and cons but it is still widely used in monopropellant thruster. Here, we bring a critical discussion on the silver catalyst as most of its weaknesses stated might has anything to do with the porosity effect in the thruster. In this study, silver is chosen because the model is adopted from Othman's et al., [20] and Othman's [32] work which used similar type of catalyst. Nevertheless, according to Pędziwiatr et al., [25] silver is considered as one of most effective heterogeneous catalysts for hydrogen peroxide decomposition.

Porosity of the catalyst medium of the monopropellant thruster give effects to the pressure drop of across the catalyst bed. The porosity and the void fraction of the bed (ratio of free volume to the absolute volume of the bed) is not coincided in the terminologies where porosity is an adjustable parameter which might not be equivalent to the real porosity of the materials; even they are assumed to possess similar order of magnitude [33]. Božic et al., [26] stated that there are two main operational indicators for the combustion. Firstly, pressure drop across catalyst bed, and secondly, total pressure drops across the feed line, where both of them have huge effect on the decomposition process stability and structure weight. Hence, pressure drop across the catalyst bed is an important parameter since it will induce the changes in performance variables such as exit pressure, exit velocity, and thrust force. Even though both pressures drops are important parameters, but only the later affected by the porosity of the catalyst. Plus, the relationship between the pressure drops and flow rate with regard to the fluid flow through porous media is due to the nature of flow through the porous media itself [34]. 
There are two main factors that influence the porosity of the catalyst bed, i.e., permeability (viscous resistance term) and inertial resistance term. Viscous friction forces contributed to the pressure drop in the laminar flow regime, while inertial forces contributed to the pressure drop in the turbulent flow regime [35]. However, Kołodziej et al., [35] stated that pressure drop across the catalyst greatly dependent on the screen type and separate correlations (between pressure drop and catalyst's porosity) are necessary for each type of screen used. Soares et al., [36] also stated that dependency and effects of viscous and inertial resistances are complex but both resistances are greatly associated to the geometrical structures of the porous material. These factors are results of the geometrical features of the catalyst such as type of catalyst used (screen, powder, pallet), compaction pressure, diameters of the catalyst, and structure (isotropic or anisotropic shape).

In this study, the main challenge was to simulate the complex flow physics and chemical reactions that may be validated with actual or experimental values. The effects of porosity of the silver catalyst for the hydrogen peroxide monopropellant thruster will be investigated and observed, and has become the objective of this study. Though, report on the effect of silver catalyst porosity in a monopropellant thruster performance are scarce, making a one to one comparison in term of relationship with the thruster performances more difficult. However, with this study, a preliminary view on this field, specifically on the effect of hydrogen peroxide monopropellant thruster with silver catalyst, will be established.

\section{Methodology}

This study is concentrating on the effect of porosity of the catalyst bed through numerical analysis. The structure or the work flow in establishing this work is shown in Figure 4 of the research flow chart. The overall process can be divided into three parts where in the first part, pre-processing section is done. In this section, the model needs to be set up together with the first meshing generation. Then, numerical solution such as setting up boundary condition, discretization schemes, initialization is carried out. Once a constant result is established after many runs, a validation process is done to see if the simulation results is validated with the experimental data. Finally, post processing which include visualization process is done to showcase the finding of this study.

Initially, the geometry of the thruster design is selected based on literature review. The design used in this study is based on adaptation from Othman [32] due to the similarity in term of thruster class and the concentration of hydrogen peroxide solution used. The design of the thruster is depicted in Figure 5. The structure of the silver catalyst used is a woven screen type which is illustrated in Figure 6, showing the structure of one ply of the screen while the actual catalyst used is formed by staking multiple plies of the screens and compacted into single shell with respect to the diameter and length of the catalyst bed. This significantly increases the complexity of the overall geometry. To simulate this section in three-dimensional (3D) has become a very challenging aspect as the structure will be astronomically difficult to perform the meshing. Later, the model is created in two-dimensional (2D) structure. 2D model is used as it is simpler and required less and faster iterations which will reduce the overall computational cost. The specifications for the thruster combustion chamber are described in Table 1 while the 2D configuration is already shown in Figure 2. For this study, all dimensions are fixed while the porosity value is varied at range of 0.2 to 0.8 . 


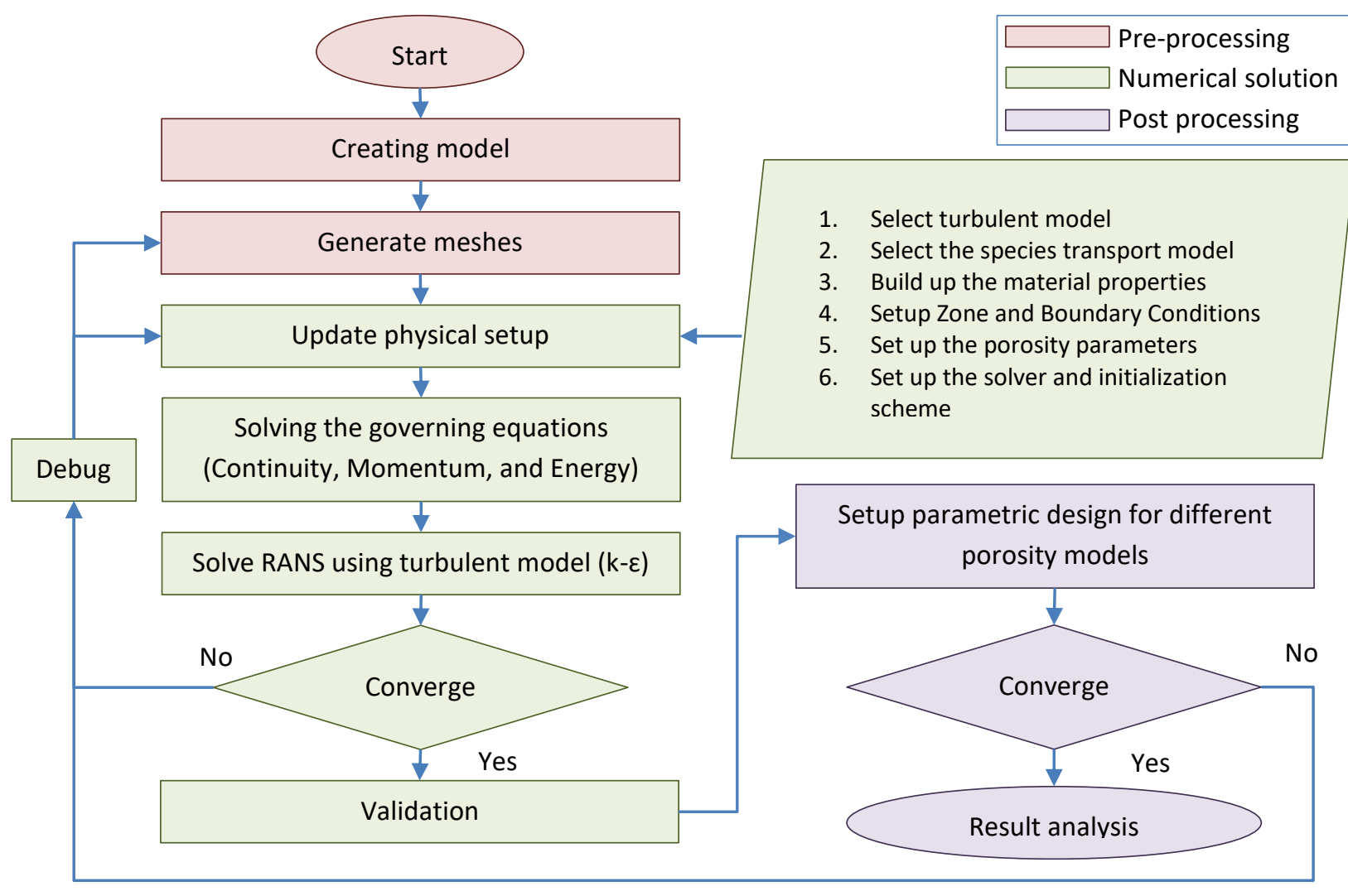

Fig. 4. Research flowchart

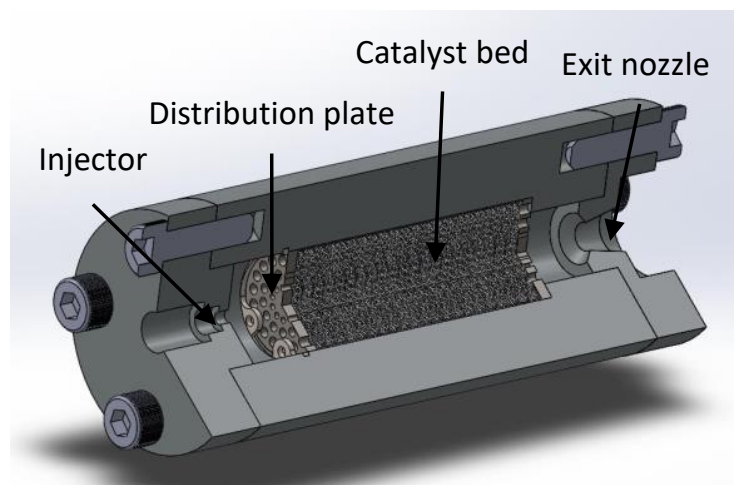

Fig. 5. Sectional view of hydrogen peroxide mono-propellant thruster

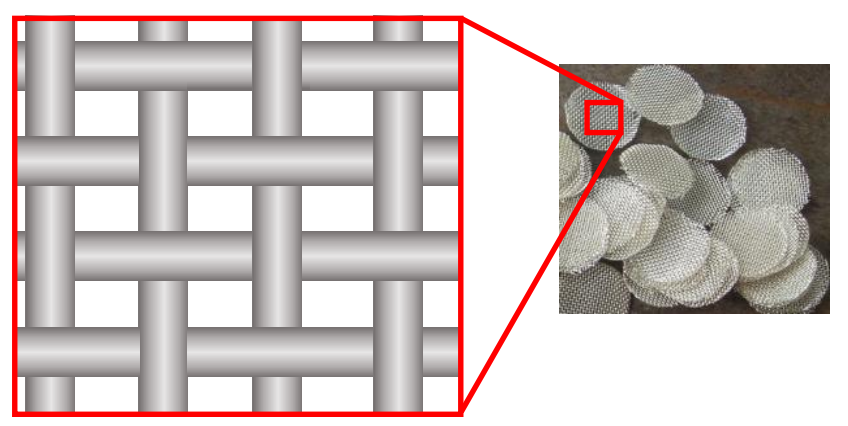

Fig. 6. Silver screen cut into circular shape. On the left illustrating the woven structure of the screen

Table 1

Thruster model specifications

\begin{tabular}{ll}
\hline Parameter & Value \\
\hline Injector diameter & $2.60 \mathrm{~mm}$ \\
Catalyst bed diameter & $17.00 \mathrm{~mm}$ \\
Catalyst bed length & $50.00 \mathrm{~mm}$ \\
Nozzle throat diameter & $4.50 \mathrm{~mm}$ \\
Nozzle exit diameter & $7.40 \mathrm{~mm}$ \\
\hline
\end{tabular}




\subsection{Grid Independent Test}

The integrity of the solution should not be affected by the mesh size. This situation can be attained by doing the grid independent test where by increasing the number of elements of the mesh, the results should maintain to proof that the result is independent from the mesh regardless how fine the grid is. The result should represent mostly of the boundary conditions and physics applied into the solution. From the test made, one size of mesh can be used if it seems sufficient and good enough to generate the converging and convincing result with consideration of the computing cost i.e., time consumed. This test is conducted to select the best base mesh size to predict the reaction event as well as for validating the simulation [37].

Three sizes of mesh have been generated with increasing number of elements from one to another. The detail of the meshes are depicted in Table 2 where all of the meshes went through the same procedure with same meshing techniques. The mesh shows in Figure 7 created by using uniform quadrilateral grid and cut into half as the thruster is symmetrical to reduce number of elements. The size of the grid for each set of Mesh are varied. As an example, for Mesh 1, the smallest grid is $0.20 \mathrm{~mm}$ and the largest grid is $1.5 \mathrm{~mm}$. The combination of the multiple grid sizes makes up the total number of elements to be 153,116. Similarly, for Mesh 2 and Mesh 3 where the mesh grid sizes range from $0.10 \mathrm{~mm}$ to $1.00 \mathrm{~mm}$ and $0.10 \mathrm{~mm}$ to $0.50 \mathrm{~mm}$, producing 368,870 and $1,209,482$ total elements respectively. As we can see, the coarseness of the mesh decreased by each iteration.
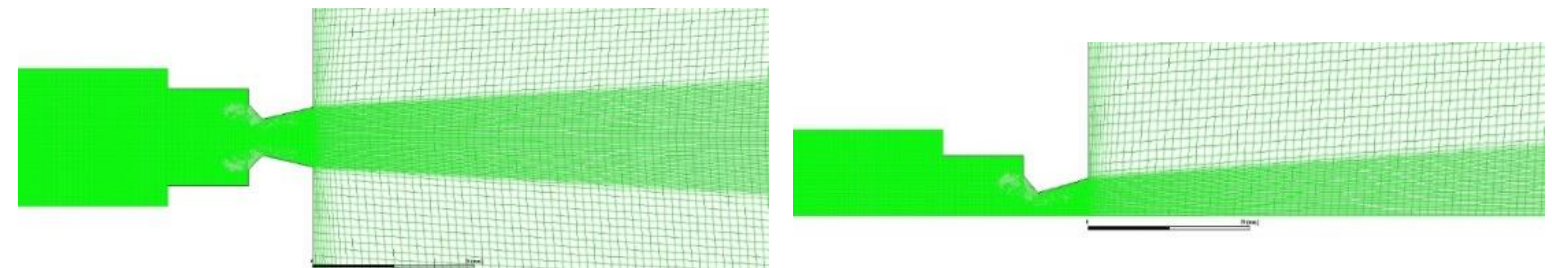

Fig. 7. 2D model of axisymmetric mesh with quadrilateral grid, left figure showing the overall mesh while the right figure showing the axisymmetric half model

Table 2

Grid independent test marks

\begin{tabular}{llllllll}
\hline Mesh & Number of & \multicolumn{2}{l}{ Element size range $(\mathrm{mm})$} & \multicolumn{4}{l}{ Quality attributes (average value) } \\
& elements & Min & Max & Element & Aspect ratio & Skewness & Orthogonal \\
\hline Mesh 1 & 153,116 & 0.20 & 1.50 & 0.956 & 1.3325 & $8.87 \mathrm{e}-003$ & 0.99967 \\
Mesh 2 & 368,870 & 0.10 & 1.00 & 0.952 & 1.2584 & $1.59 \mathrm{e}-002$ & 0.99916 \\
Mesh 3 & $1,209,482$ & 0.10 & 0.50 & 0.973 & 1.1113 & $1.69 \mathrm{e}-002$ & 0.99938 \\
\hline
\end{tabular}

From these meshes, the quality attributes seem reasonable where the value of aspect ratio for all meshes are greater than 1 while the average skewness of each grid cell is very small. This mean that most of the cell created are closed to the ideal mesh geometry such as equilateral or equiangular which have skewness value equal to zero [38]. Furthermore, elements with orthogonal quality of 0.95 to 1 was considered to be excellent [39]. Hence, based on these attributes the quality of all meshes created is validated.

From the grid independent test done, all meshes results in a small difference. This is shown in the temperature distribution in Figure 8, where the temperature variation is recorded at the center of the thruster showing that maximum temperature measured at the catalyst bed are $1120 \mathrm{~K}$ for Mesh 1 and $1164 \mathrm{~K}$ for both Mesh 2 and Mesh 3. By comparing the computing cost for each mesh in Table 3 , the first mesh can produce a result at speed of 0.963 second of the wall-clock time per iteration, while the second and third run at speed of 2.285 and 6.753 seconds. After comparing all three results 
from this test, second mesh is chosen as a basis of this study. Considering that the speed to achieve stable result is slightly longer than the first mesh but not as slow as the third mesh and most importantly it is in compliment with the consistent maximum temperature achieved by the simulation which shown in the second and third mesh. A conclusion could be made that even though the number of elements expending and the grid sizes became finer, but the results does not show significant effects except that it increases the number of iterations for each solution. Hence, by considering the computational cost and number of iterations needed to attained the convincing and converging results, Mesh 2 is then used for this study.

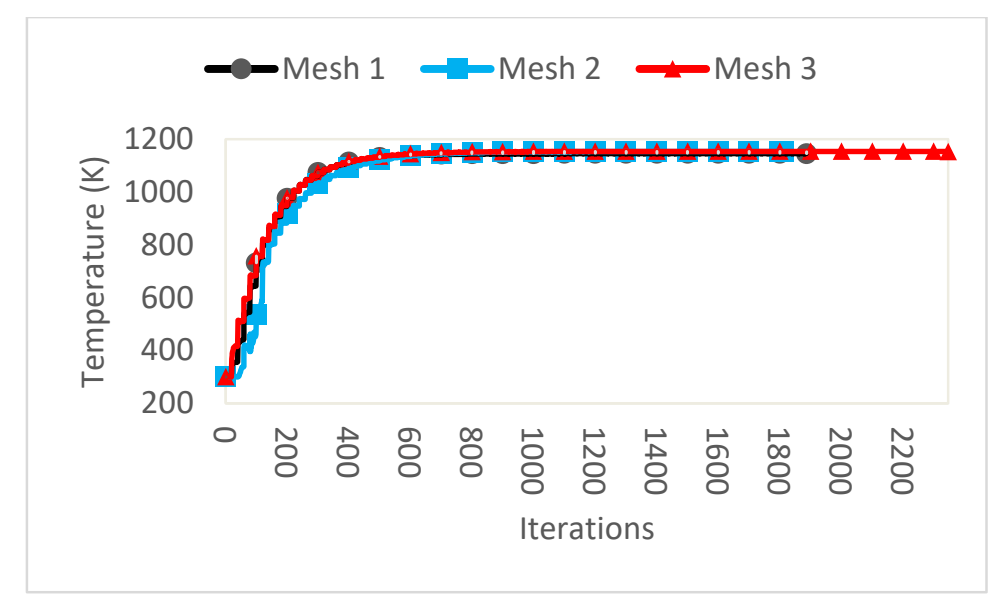

Fig. 8. Temperature plots for three different meshes

\section{Table 3}

Duration for each meshes to reach convergence criteria

\begin{tabular}{lllll}
\hline Mesh & Number of Iterations & $\begin{array}{l}\text { Total wall-clock time } \\
(\mathrm{s})\end{array}$ & $\begin{array}{l}\text { Average wall-clock } \\
\text { time per iteration (s) }\end{array}$ & $\begin{array}{l}\text { Maximum temperature } \\
\text { at catalyst bed (K) }\end{array}$ \\
\hline Mesh 1 & 1886 & 1817 & 0.963 & 1120 \\
Mesh 2 & 1814 & 4144 & 2.285 & 1164 \\
Mesh 3 & 2347 & 15848 & 6.753 & 1164 \\
\hline
\end{tabular}

\subsection{Governing Equations}

The flow physics and reactions inside the thruster simulation are governed by the conservation of mass, momentum, and energy. The continuity equation which depicted in Eq. (2) shows the mass conservation law with additional isotropic porosity in a single-phase flow where $\gamma$ represent the porosity of the catalyst bed. While $\rho, t$, and $\vec{v}$ represent density, time, and velocity vector.

$$
\frac{\partial(\gamma \rho)}{\partial t}+\nabla \cdot(\gamma \rho \vec{v})=0
$$

Eq. (3) shows the momentum conservation equation where momentum source term, $S_{i}$, has been added to the standard fluid flow equation. The momentum source term, which represented by Eq. (4) is the part that influenced by the porosity of the catalyst bed; where this function contributes to the pressure gradient in the porous region which led to the pressure loss. In Eq. (4), the first term of the right-hand side represents a viscous loss term, while the second term showing the inertial loss term, $C_{2}$. Viscous resistance term, $1 / \alpha$, is also a function of inverse absolute permeability; where permeability, $\alpha$, is defined as a characteristic of the porous medium which calculates the capacity 
and ability of the structure to transmit fluids. The turbulent stress tensor, $\overline{\bar{\tau}}$ in Eq. (3) is expended further in Eq. (5), where $\mu$ is a viscosity of the fluid, and $I$ is the unit tensor.

$$
\begin{aligned}
& \frac{\partial}{\partial t}(\rho \vec{v})+\nabla \cdot(\rho \vec{v} \vec{v})=-\nabla p+\nabla \cdot(\overline{\bar{\tau}})+\rho \vec{g}+S_{i} \\
& S_{i}=-\left(\frac{\mu}{\alpha} v_{i}+C_{2} \frac{1}{2} \rho|v| v_{i}\right) \\
& \overline{\bar{\tau}}=\mu\left[\left(\nabla \vec{v}+\nabla \vec{v}^{T}\right)-\frac{2}{3} \nabla \cdot \vec{v} I\right]
\end{aligned}
$$

The conservation of energy is represented in Eq. (6) with additional energy source due to chemical reaction, $S_{h}$. In this equation, $k_{e f f}$ is the effective conductivity; it is a product of turbulent and turbulent thermal conductivity which will be defined according to the selected turbulence model. The first term of the right-hand side of Eq. (6) represents the energy transfer due to conduction, the second term represents the species diffusion, and the third term represents the viscous dissipation, while, $\vec{J}_{j}$ is the diffusion flux of the species. The energy source due to chemical reaction expended in Eq. (7) which includes the volumetric heat sources without the heat sources generated by the finiterate (volumetric or surface) reactions. In Eq. (7), $h_{j}^{0}$ represents the enthalpy of the species while $\mathfrak{R}_{j}$ is the volumetric creation rate of species. Eq. (8) shows the species transport equation where $Y_{i}$ represents the local mass fraction of the species, $R_{i}$ is the species production rate through chemical reaction, and $S_{k}$ is the creation rate.

$$
\begin{aligned}
& \frac{\partial}{\partial t}(\rho E)+\nabla \cdot(\vec{v}(\rho E+p))=\nabla \cdot\left(k_{e f f} \nabla T-\sum_{j} h_{j} \vec{J}_{j}+\left(\overline{\bar{\tau}}_{e f f} \cdot \vec{v}\right)\right)+S_{h} \\
& S_{h}=-\sum_{j} \frac{h_{j}^{0}}{M_{j}} \Re_{j} \\
& \frac{\partial}{\partial t}\left(\rho Y_{i}\right)+\nabla \cdot\left(\rho \vec{v} Y_{i}\right)=-\nabla \cdot \overrightarrow{J_{i}}+R_{i}+S_{k}
\end{aligned}
$$

\subsection{Setup Procedure}

The meshed model first set up with complete boundary conditions and zone conditions as depicted in Figure 9. The boundary condition generally consists of pressure inlet, pressure outlet, adiabatic walls with zero heat flux conditions, and axisymmetric axis. Catalyst bed region is set to be porous medium with velocity formulation is set to be superficial. The reaction is enable considering that decomposition of hydrogen peroxide only occurs in the catalyst bed. While inlet is supplied with hydrogen peroxide-water-air mixture material and the mass fraction of the inlet is set to have 0.9 of hydrogen peroxide and 0.1 mass fraction of water. The mass fraction ratio selected is corresponding to the $90 \%$ hydrogen peroxide concentration used in this study, with assumption that the solution be without any trace of impurities including stabilizers or any additives that typically presence. The inlet pressure and temperature values are fixed at $2.96 \mathrm{MPa}$ and $300 \mathrm{~K}$ respectively.

In this study, steady state pressure-based solver is used. Standard $k-\varepsilon$ turbulent model is selected and standard wall functions for near-wall treatment opted. Energy equation is activated. In species 
transport option, volumetric reaction is activated. The turbulent-chemistry interaction utilized the Eddy Dissipation Model (EDM) considering that reaction rates are assumed to be controlled by the turbulence. The input parameters are shown in Table 4. Solution methods used to simulate this setup is by using COUPLED Scheme with hybrid initialization. In this study the porosity of the catalyst bed is varied from 0.2 to 0.8 .

Few simplifications and assumption had to be made with careful justification. Vaporized hydrogen peroxide was assumed to be in gas phase that are subjected to the kinetic theory of molecules. Other assumptions made are, the catalyst used is considered isotropic and the axial heat conduction is disregard, the impurity in the hydrogen peroxide solution is neglected, the radiation heat transfer is ignored, and the only product of the reaction are water vapor and oxygen.

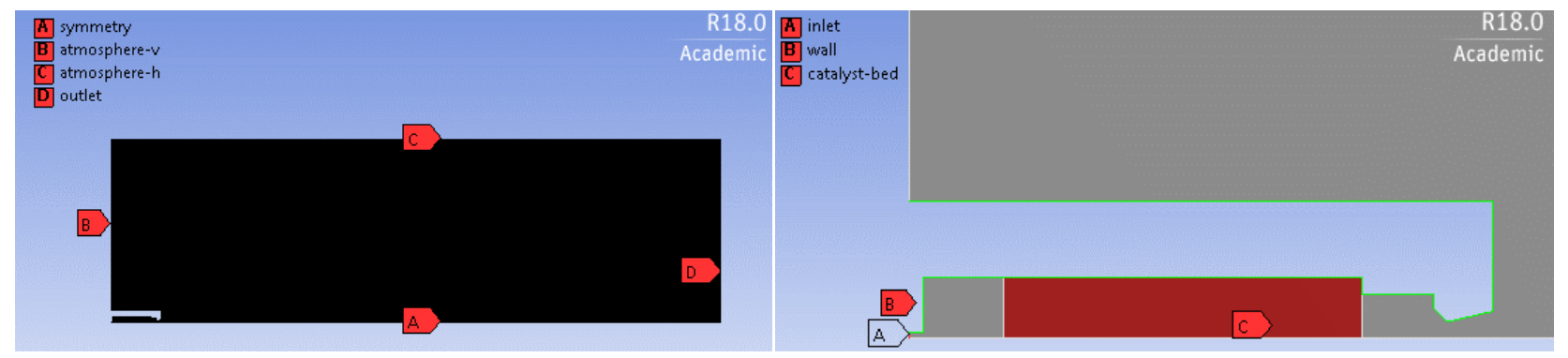

Fig. 9. Boundary condition of the model including porous medium zone condition (shaded)

Table 4

\begin{tabular}{ll} 
Input parameter for flow physics & \\
\hline Parameter & Value \\
\hline Inlet absolute pressure & $2.96 \mathrm{MPa}$ \\
Inlet temperature & $300 \mathrm{~K}$ \\
Concentration of Hydrogen Peroxide & $90 \mathrm{wt} \%$ \\
Operating pressure & $0.101 \mathrm{MPa}$ \\
Porosity & $0.2-0.8$ \\
Viscous resistance & $5.67 \mathrm{e}+09 \mathrm{~m}^{-2}$ \\
Inertial resistance & $6.09 \mathrm{e}+04 \mathrm{~m}^{-1}$ \\
\hline
\end{tabular}

\section{Results}

The simulation of the hydrogen peroxide monopropellant thruster is performed with several iterations on different values of catalyst pack porosity ranging from 0.2 to 0.8 . Initially, single case of simulation is done to validate the result in terms of the precision of the simulation result to the real scenarios. Few variables can be compared, and in our case, the catalyst bed temperature is compared with the experimental data; and the species mass fraction are compared with the theoretical values, to make sure the simulation result is justified. However, other parameters such as Mach number, densities, and velocities, that the experiment data may available can be used as a relative or one-toone comparison with the simulation.

In this simulation temperature reached highest value of slightly more than $1100 \mathrm{~K}$. From this, it is safe to say that temperature inside the catalyst bed can go from 700K up to more than $1000 \mathrm{~K}$ which is compliment with the experimental findings such as by work of Othman [32], Kuan et al., [15], and Jung et al., [18] that acquired temperature of $>700 \mathrm{~K}$ to $1020 \mathrm{~K}$ for the $90 \mathrm{wt} \%$ hydrogen peroxide. Figure 10 show the temperature different between experimental work adopted and the simulation result [32]. The different of $13.7 \%$ is calculated. Different in those results are due to in experiment, the presence of impurity cannot be removed hundred percent while in simulation, hydrogen peroxide 
is in a pure condition. Plus, the measurement in the experiment may exposed to many challenges such as the effect of ambient temperature and pressure, thermostat calibration, as well as other factors that might contributes to the results. Temperature distribution in the thruster is shown in Figure 11. From this figure, the temperature contour shows that heat is concentrated in the catalyst bed area. After the decomposition process take place inside the catalyst bed region, the flow brings out the heat from the reaction through nozzle. Then, the heat outside the catalyst bed mixed with the ambient air and equilibrates with the surrounding and started to decrease as it moves further from the reaction site.

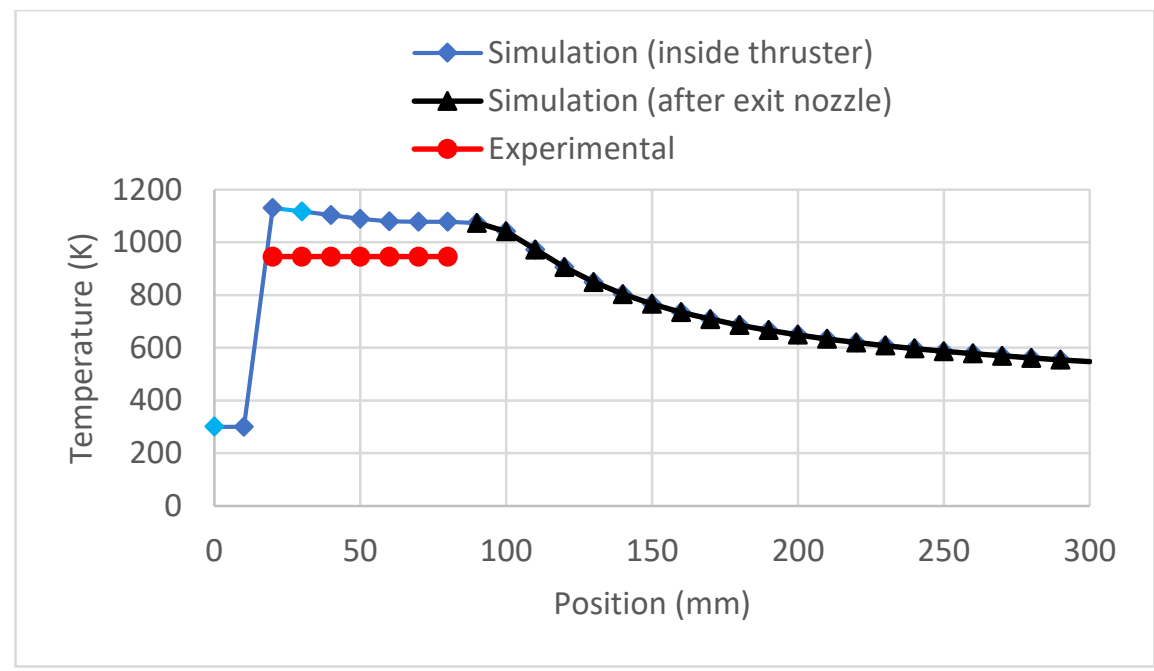

Fig. 10. Temperature distribution

From Figure 12, the mass fraction of $\mathrm{H}_{2} \mathrm{O}_{2}, \mathrm{H}_{2} \mathrm{O}$, and $\mathrm{O}_{2}$ clearly demonstrates the dissociating of hydrogen peroxide into water vapor and oxygen gas where hydrogen peroxide started at 0.9 mass fraction dropped significantly once it reached the catalyst bed. This shows that the reaction took place as the mass fraction of oxygen gas started to form, while the mass fraction of water vapor rises. Mass fraction of hydrogen peroxide continuously depleted in exchange with the water vapor and oxygen gas that rose until they both reached maximum stoichiometric mass fraction. Both species then mixed with ambient air once released through the nozzle together with the remaining of hydrogen peroxide that are approaches zero mass fraction. Water vapor mass fraction started to decline outside the thruster and the oxygen gas mass fraction also went down approaching 0.23 as a standard mass fraction of oxygen in air.

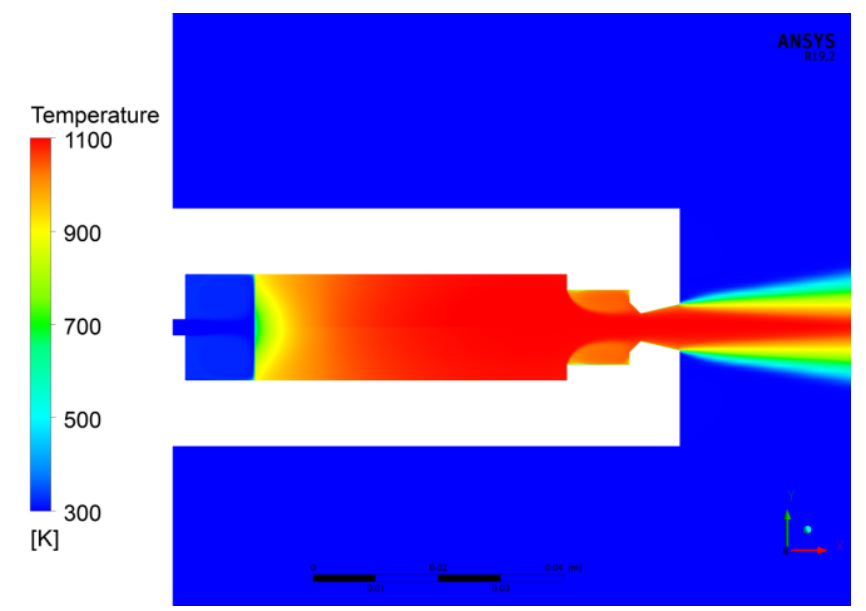

Fig. 11. Temperature contour inside thruster

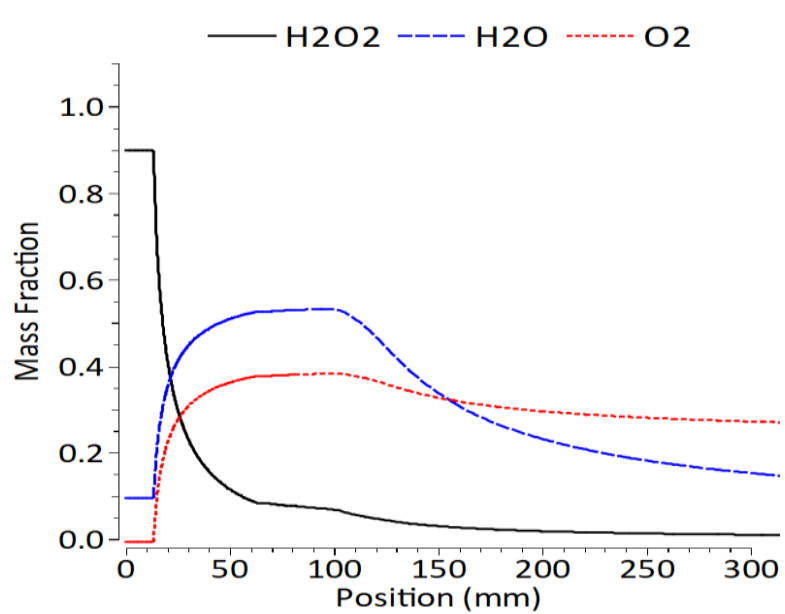

Fig. 12. Species mass fraction across thruster 
Species mass fraction graph and the temperature contour if mapped together showing that the flow has a correct decomposition reaction and heat generation from the reaction. Hence, this justified that the simulation is producing reliable result. On the other hand, other parameters can also be observed, as a precaution and double confirming the solution of the simulation.

The velocity of the flow inside thruster is shown in Figure 13. In this figure, the velocity contour shows that at the throat of the nozzle, the velocity is forced to increase as the catalyst bed continuously supplying the flow with pressure while the throat area was narrowed. The different values of velocities can be seen throughout the thruster except for the catalyst bed region. In that region, the velocity contour shows uniformity as it relatively not accurate and does not depict the actual physical phenomenon that happened inside the catalyst bed. However, it is acceptable and for this simulation, it was expected to behave in such way as that region is assumed to be isotropic as to ensure the continuity of the flow velocity, and to simplify the simulation process. In reality, most of the catalyst materials are made from screen, pallet, and powder where these types of shape typically closer to anisotropic geometry rather than being isotropic. However, anisotropic of the catalyst bed is hardly known (or calculated) due to irregular geometry of the said catalyst materials unless in some cases where specific type of shape is used to make the catalyst medium such as monolith cylinders or honeycomb. In recent development, this shape is easier to be made with 3D printer or SLS technology and will help in determining the exact value of anisotropic parameter. The same difficulty is faced when determining the values of viscous and inertial resistance; due to irregularity catalyst bed shape. These values can be calculated using few techniques such as using Darcy's or Ergun's formulation. However, most cases are unique and different from one to another, so the best way to determine these values are through experiment data by comparing the polynomial trend of the pressure drop across porous medium versus the velocity.

From Figure 14, total pressure contour across catalyst bed is shown. From this figure, it is clear that the pressure magnitude dropped across the catalyst bed region. Pressure lost is measured at range of $2.8 \mathrm{MPa}$ for all different porosity value. Pressure drop across catalyst bed (denoted as $\Delta \mathrm{P}_{\mathrm{CP}}$ ) varies with different porosity values ranges from 0.2 to 0.8 shown in Figure 15, depicting the relationship between pressure drop and porosity of the silver catalyst. Pressure drop is measured by finding the different of pressures at two locations. One which are located before the catalyst bed region and another one just right after it.

As the catalyst porosity increases, the pressure drops across the catalyst bed also increases until reached maximum pressure drop before deflected and went down as the porosity increases. The inclination and declination are quite subtle but still showing the significant of the porosity value to the pressure drop. However, porosity alone is not a sole criterion in influencing the pressure drop, nevertheless, it still has an impact to the flow behaviour. This can be seen in Figure 16, where the effects and influence of the porosity emerges in terms of the velocity as well. The velocity mentioned here is the exit velocity, which mean the velocity of the flow measured at the nozzle exit of the thruster. The figure shows an interaction between porosity and velocity where with increased porosity, velocity is reduced almost linearly. 


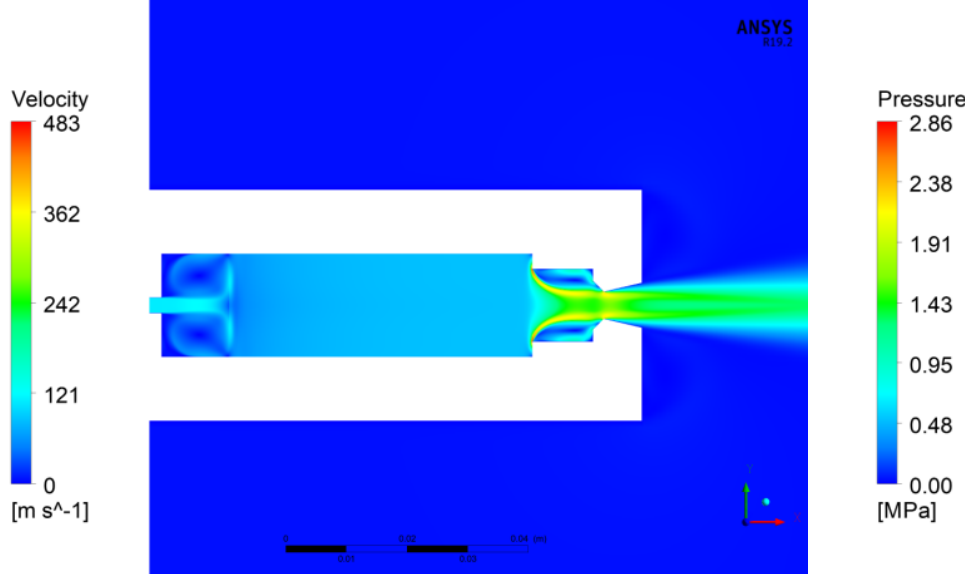

Fig. 13. Velocity contour

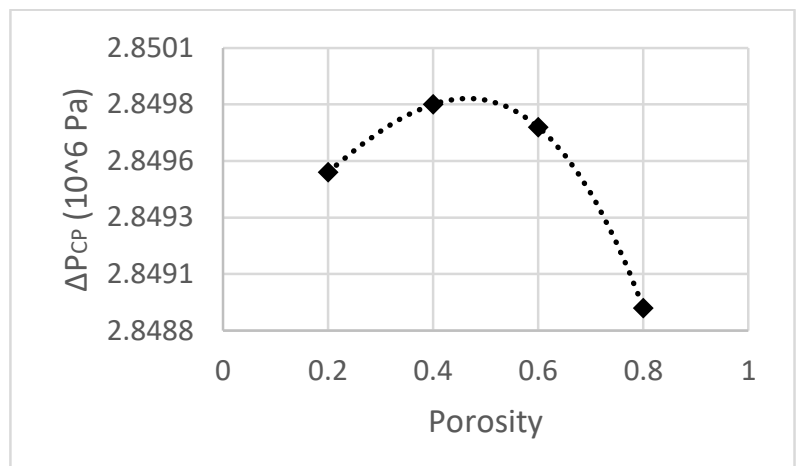

Fig. 15. Graph of pressure drop across catalyst bed against silver catalyst porosity

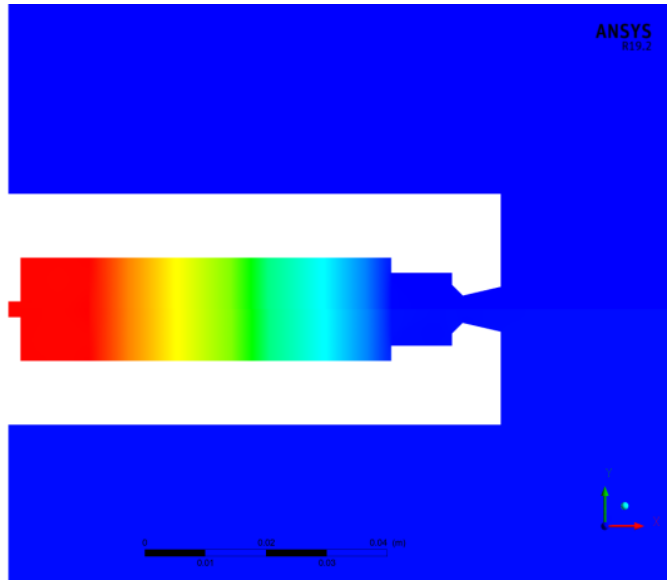

Fig. 14. Pressure contour

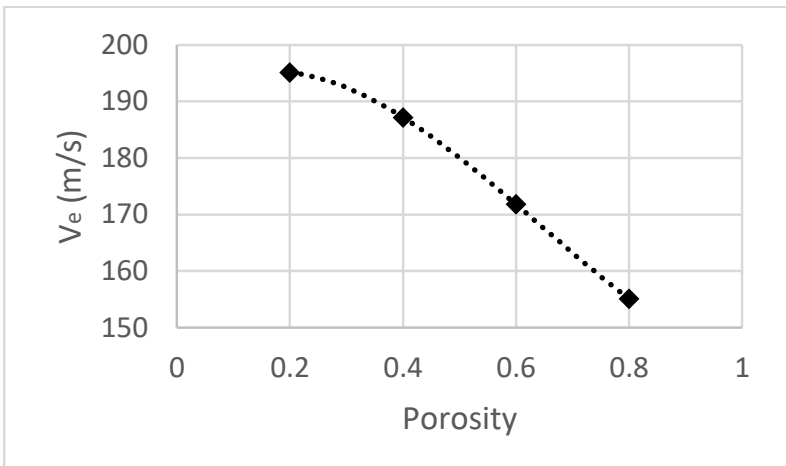

Fig. 16. Graph of exit velocity against silver catalyst porosity

Porosity of the catalyst bed may undergo changing throughout the entire reaction due to the fact that catalyst bed may be physically altered as an effect from the reaction itself; as discussed in the previous section, catalyst attrition, channelling, or sintering might happen where it will eventually disrupt the initial original porosity of catalyst. So, during the reaction take place, the porosity may have changes throughout the entire period of reaction. This will lead to a fluctuation of the pressure drop across the catalyst bed. However, in this study, the changes of the porosity are somehow quite static while changes of porosity of the catalyst during the reaction can be more dynamic and vigorous. Nonetheless, the effect of the porosity to the pressure drop still can be observed. A postulation could be made by extrapolating the behaviour of changing porosity at greater rate, so the pressure loss fluctuates at certain frequency can be presumed to be in higher order of magnitude, creating an oscillating behaviour can be recorded.

The pressure oscillation inside the thruster chamber can affect other behaviour of physical phenomenon such as unsteady exit velocity, and unsteady thrust force. Other concern about this behaviour is that the pressure oscillation can influence the structural strength of the thruster body since it will be hit with recurring waves of pressures and may inducing vibration noise, in addition to the weakening effect by the thermal stress of such high temperature produces during the reaction process. This may be affecting the stability and life span of the thruster body itself. As the case was run in steady state mode instead of transient, the pressure oscillation cannot be observed or measured.

As the porosity of the catalyst bed increases, the structure of the catalyst medium supposedly be more packed and tighter. This mean that the flow will have to overcome a greater resistance in the 
flow field as it needs to travel through smaller channel, this will eventually result in the difference of pressure.

Pressure drop is a parameter that can be related to the permeability, $\alpha$ (where in this article, we use the inverse permeability or also viscous resistance, denoted by $1 / \alpha$ ) and inertial resistance, $C_{2}$ as described in Eq. (4) in Section 2.2 Governing Equations. Both parameters are the function of geometry of the porous material.

The relationship between pressure drops and porosity of the catalyst bed is associated with the mass conservation as described in the Eq. (2) where porosity, $\gamma$ was introduced into the continuity equation. The porosity affects few parameters such as velocity and pressure, however, as described in Eq. (3), the porosity of the catalyst bed has become a contributing factor to the additional momentum source (sink), $S_{i}$ into the momentum equation, and hence, influencing the pressure drop inside the bed.

The momentum source described there consist of permeability and inertial resistance factors. These parameters can be solved using 1D or 2D solution, where in this study these parameters are solved using 2D Cell Zone solution instead of 1D porous jump boundary condition. In this study, these two terms were fixed as it is considered beyond the scope of this article as only porosity parameter is varied. Still, the effect of the porosity can be observed in few areas.

The temperature inside the catalyst bed changes with porosity in a second or third order of polynomial relationship. Temperature increases as the porosity increased until it reached maximum temperature of slightly $>1160 \mathrm{~K}$ when porosity at 0.4 to 0.5 , then, when the porosity continues to increased, the temperature inside the thruster chamber starting to fall again. This behaviour is recorded in Figure 17, showing the temperature reached against different porosity values. The behaviour of the temperature versus catalyst bed porosity is similar to that of pressure drop across catalyst bed. From both graphs, we can deduce that porosity ranging from 0.4 to 0.6 producing maximum temperature and pressure drop. While exit velocity and thrust force showed a similar pattern; no significant porosity value can be seen since both showed a negative gradient against increasing porosity. Thrust force values against catalyst porosity depicted in Figure 18, showing that thrust force ranging from approximately $156 \mathrm{~N}$ to $150 \mathrm{~N}$.

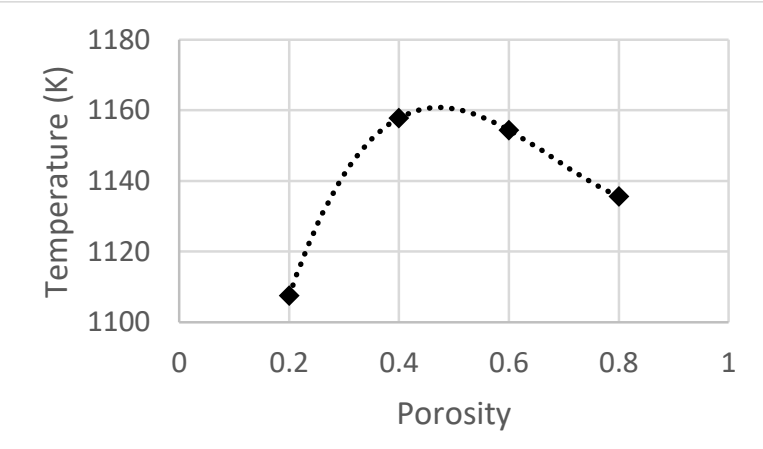

Fig. 17. Graph of catalyst bed temperature against silver catalyst porosity

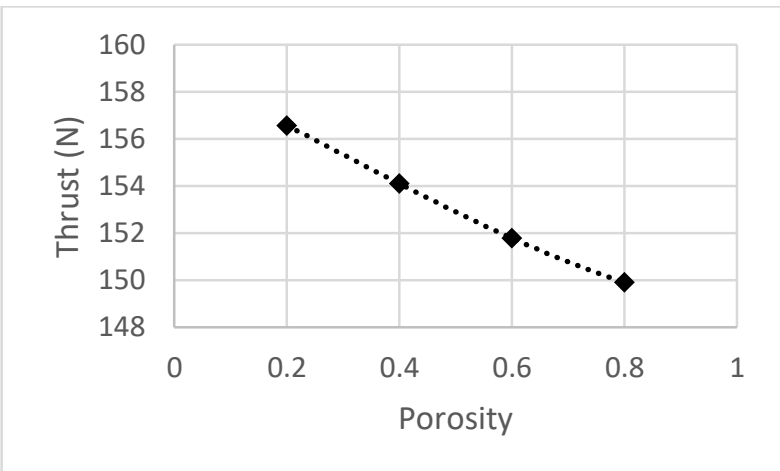

Fig. 18. Graph of thrust force against silver catalyst porosity

\section{Conclusions}

The effect of the silver catalyst porosity towards the hydrogen peroxide monopropellant thruster performances rarely gets attention as most of the researches done experimentally, which is hard to specifically control the porosity value. Hence, the optimum porosity needed is quite uncertain. An optimized porosity value for the silver catalyst bed will help in reducing the impact of silver catalyst's 
drawbacks. As discussed in Introduction section, silver catalyst faces problem such as catalyst attrition, sintering, and channelling where these problems might be reduced when a correct porosity is selected. Even though it might not solve the problems completely, it is still wise to know and understand the effect of the porosity when designing the hydrogen peroxide monopropellant thruster with silver catalyst. It is also important in tuning the performance by leveraging the porosity in developing a precise thruster control.

To investigate the influence of the porosity and to find the optimal value, a single-phase simulation is done. By using steady state solver, and standard $k-\varepsilon$ turbulent model, the simulation is made by varying the porosity value from 0.2 to 0.8 . Due to constraint such as limited number of literatures, the finding of this study cannot be compared to know the extent of its accuracy. Besides, in order to reduce the computational cost and speed up the study, simulation is made with many simplifications. The decomposition reaction inside the catalyst bed fit the definition of multiphase model where the flow is considered multiphase if more than one state of material presence during the reaction take place. In our case the liquid-gas flow in the decomposition reaction is reduced to gas reaction. This we believed somehow affecting the result of our study in term of its accuracy, still, the overall sentiment of the result does not go against us because other indicator such as temperature profile, mass fraction plot, and other parameter still parallel with the results of the experiment adopted. Nevertheless, this is still preliminary and hopefully many works in the future will be discussing this matter and improving the finding. To further increase the credential and accuracy of this result, a multiphase model simulation should be developed.

In addition to that, porous medium characteristic of the silver catalyst impose additional momentum source due to the permeability and inertial resistance. However, these two terms still cannot be assessed as this study is only focus on the effect of porosity. Even though porosity and permeability and inertial resistance do not dependent on each other but permeability and inertial resistance is a derivative of the porous medium itself. However, the link or relationship of those parameters with porosity of the silver catalyst is unknown especially in our case of the hydrogen peroxide decomposition via porous silver catalyst. A relationship between porosity of these two parameters should be investigated; especially in the hydrogen peroxide decomposition process.

Conclusively, it found that the porosity of 0.4 to 0.5 is an optimal porosity value that shows a maximum performance in temperature and pressure drop. While for the thrust force and exit velocity, performance are decreases as the porosity value increases. Ergo, this study managed to provide mainly the effect of the porosity to the pressure drop, exit velocity, catalyst bed temperature, and thrust.

\section{Acknowledgement}

This study is part of the work on hydrogen peroxide monopropellant thruster development and optimization program at the Liquid Propulsion Laboratory, School of Mechanical Engineering of the Universiti Teknologi Malaysia. It was supported by the Ministry of Higher Education with Fundamental Research Grant Scheme (award No. 5F099). Much thanks and appreciations to the school, and university in providing the facilities needed in conducting this research.

\section{References}

[1] Salgado, Maria Cristina Vilela, Mischel Carmen Neyra Belderrain, and Tessaleno Campos Devezas. "Space propulsion: A survey study about current and future technologies." Journal of Aerospace Technology and Management 10 (2018). https://doi.org/10.5028/jatm.v10.829

[2] Meyer, Michael, Les Johnson, Bryan Palaszewski, David Coote, Dan Goebel, and Harold White. Roadmap for inspace propulsion technology. No. NASA/TM-2012-217641. Glenn Research Center, Cleveland (Ohio), 2012. 
[3] Rhodes, Brandie L., and Paul D. Ronney. "Dynamics of a Small-Scale Hydrogen Peroxide Vapor Propulsion System." Journal of Propulsion and Power 35, no. 3 (2019): 595-600. https://doi.org/10.2514/1.B37323

[4] Jang, Dongwook, Shinjae Kang, and Sejin Kwon. "Preheating characteristics of H2O2 monopropellant thruster using manganese oxide catalyst." Aerospace Science and Technology 41 (2015): 24-27. https://doi.org/10.1016/i.ast.2014.12.010

[5] Monopropellant rocket engines. 2 August 2021

[6] Chan, Y. A., H. J. Liu, K. C. Tseng, and T. C. Kuo. "Development and hot-firing test of a hydrogen peroxide thruster for Formosat-7 project." In Space Propulsion Conference. Cologne, Germany, 2014.

[7] Florczuk, Wojciech, and Grzegorz P. Rarata. "Performance evaluation of the hypergolic green propellants based on the HTP for a future next generation spacecrafts." In 53rd AIAA/SAE/ASEE Joint Propulsion Conference, p. 4849. 2017. https://doi.org/10.2514/6.2017-4849

[8] Essa, Khamis, Hany Hassanin, M. M. Attallah, N. J. Adkins, A. J. Musker, G. T. Roberts, N. Tenev, and Matthew Smith. "Development and testing of an additively manufactured monolithic catalyst bed for HTP thruster applications." Applied Catalysis A: General 542 (2017): 125-135. https://doi.org/10.1016/j.apcata.2017.05.019

[9] Baek, Seungkwan, Woosuk Jung, Hongjae Kang, and Sejin Kwon. "Development of high-performance greenmonopropellant thruster with hydrogen peroxide and ethanol." Journal of Propulsion and Power 34, no. 5 (2018): 1256-1261. https://doi.org/10.2514/1.B37081

[10] Batonneau, Y., R. Brahmi, B. Cartoixa, K. Farhat, C. Kappenstein, S. Keav, G. Kharchafi-Farhat, L. Pirault-Roy, M. Saouabé, and C. Scharlemann. "Green propulsion: catalysts for the european FP7 project GRASP." Topics in Catalysis 57, no. 6-9 (2014): 656-667. https://doi.org/10.1007/s11244-013-0223-y

[11] Gohardani, Amir S., Johann Stanojev, Alain Demairé, Kjell Anflo, Mathias Persson, Niklas Wingborg, and Christer Nilsson. "Green space propulsion: Opportunities and prospects." Progress in Aerospace Sciences 71 (2014): 128149. https://doi.org/10.1016/i.paerosci.2014.08.001

[12] Sobczak, Kamil M., Pawel Surmacz, Bartosz Bartkowiak, Adam Okninski, Grzegorz P. Rarata, Piotr Wolanski, Dominik Kublik, and Ferran Valencia Bel. "Test campaign of a green liquid bi-propellant rocket engine using catalytically decomposed 98\% hydrogen peroxide as oxidizer." In 53rd AIAA/SAE/ASEE Joint Propulsion Conference, p. 4926. 2017. https://doi.org/10.2514/6.2017-4926

[13] Talib, Abd Rahum Abu, Kamarulzaman Zainal, and Iskandar Hack. "Malaysian Aerospace Industry Blueprint 2030." Malaysian Industry Government Group for High Technology (2015).

[14] Huh, Jeongmoo, Daeban Seo, and Sejin Kwon. "Fabrication of a liquid monopropellant microthruster with built-in regenerative micro-cooling channels." Sensors and Actuators A: Physical 263 (2017): 332-340. https://doi.org/10.1016/j.sna.2017.06.028

[15] Kuan, Chih-Kuang, Guan-Bang Chen, and Yei-Chin Chao. "Development and ground tests of a 100-millinewton hydrogen peroxide monopropellant microthruster." Journal of Propulsion and Power 23, no. 6 (2007): 1313-1320. https://doi.org/10.2514/1.30440

[16] Chan, Yung-An, Hung-Wei Hsu, and Yei-Chin Chao. "Development of a HTP mono-propellant thruster by using composite silver catalyst." In 47th AIAA/ASME/SAE/ASEE Joint Propulsion Conference \& Exhibit, p. 5693. 2011. https://doi.org/10.2514/6.2011-5693

[17] Rarata, Grzegorz, Karolina Rokicka, and Paweł Surmacz. "Hydrogen Peroxide as a High Energy Compound Optimal for Propulsive Applications." Central European Journal of Energetic Materials 13, no. 3 (2016): 778-790. https://doi.org/10.22211/cejem/65005

[18] Jung, Sangwoo, Sukmin Choi, and Sejin Kwon. "Design optimization of green monopropellant thruster catalyst beds using catalytic decomposition modeling." In 53rd AIAA/SAE/ASEE Joint Propulsion Conference, p. 4924.2017. https://doi.org/10.2514/6.2017-4924

[19] Shahrin, Muhammad Shahrul Nizam, Norazila Othman, and Mastura Ab Wahid. "Liquid Propellant: Catalyst Pact Pressure Drop Effect of $50 \mathrm{~N}$ Green Thruster." In 17th International Conference on Sustainable Energy Technologies - SET 2019. 2019.

[20] Othman, Norazila, Subramaniam Krishnan, Wan Khairuddin Wan Ali, and Mohammad Nazri Mohd Jaafar. "Design and testing of a 50N hydrogen peroxide monopropellant rocket thruster." Jurnal Mekanikal 33 (2011): 70-81.

[21] Cervone, Angelo, Lucio Torre, Luca d'Agostino, Antony J. Musker, Graham T. Roberts, Cristina Bramanti, and Giorgio Saccoccia. "Development of hydrogen peroxide monopropellant rockets." In 42nd AIAA/ASME/SAE/ASEE Joint Propulsion Conference \& Exhibit, p. 5239. 2006. https://doi.org/10.2514/6.2006-5239

[22] Lee, Su-Lim, and Choong-Won Lee. "Performance characteristics of silver catalyst bed for hydrogen peroxide." Aerospace Science and Technology 13, no. 1 (2009): 12-17. https://doi.org/10.1016/i.ast.2008.02.007

[23] Amri, Redha, D. Gibbon, and T. Rezoug. "The design, development and test of one newton hydrogen peroxide monopropellant thruster." Aerospace Science and Technology 25, no. 1 (2013): $266-272$. https://doi.org/10.1016/j.ast.2012.02.002 
[24] Haq, Nadeem Ul, Raheel Aziz Khan, and Rashid Mehmood. "Design, development and testing of $1 \mathrm{~N}$ Hydrogen Peroxide thruster." In 2017 14th International Bhurban Conference on Applied Sciences and Technology (IBCAST), pp. 599-607. IEEE, 2017.

[25] Pędziwiatr, Paulina, Filip Mikołajczyk, Dawid Zawadzki, Kinga Mikołajczyk, and Agnieszka Bedka. "Decomposition of hydrogen peroxide-kinetics and review of chosen catalysts." Acta Innovations 26 (2018): 45-52. https://doi.org/10.32933/Actalnnovations.26.5

[26] Božić, Ognjan, Dennis Porrmann, Daniel Lancelle, and Stefan May. "Enhanced development of a catalyst chamber for the decomposition of up to $1.0 \mathrm{~kg} / \mathrm{s}$ hydrogen peroxide." CEAS Space Journal 8, no. 2 (2016): 77-88. https://doi.org/10.1007/s12567-015-0109-x

[27] Widdis, Stephen, Darren Hitt, Kofi Asante, Michael Cross, Walter Varhue, and Michael McDevitt. "Computational and experimental studies of catalytic $\mathrm{H} 2 \mathrm{O} 2$ decomposition in microscale reactors." In 43rd AIAA Thermophysics Conference, p. 3096. 2012. https://doi.org/10.2514/6.2012-3096

[28] Gagne, Kevin R., Darren L. Hitt, and Michael R. McDevitt. "Development of an additively manufactured microthruster for nanosatellite applications." In 54th AIAA Aerospace Sciences Meeting, p. 0963. 2016. https://doi.org/10.2514/6.2016-0963

[29] Khaji, Zahra, Lena Klintberg, Kristoffer Palmer, and Greger Thornell. "Catalytic effect of platinum and silver in a hydrogen peroxide monopropellant ceramic microthruster." Propulsion and Power Research 9, no. 3 (2020): 216 224. https://doi.org/10.1016/i.jppr.2020.08.003

[30] Kang, Hongjae, Dahae Lee, Shinjae Kang, and Sejin Kwon. "Effect of $\mathrm{H} 2 \mathrm{O} 2$ injection patterns on catalyst bed characteristics." Acta Astronautica 130 (2017): 75-83. https://doi.org/10.1016/i.actaastro.2016.10.023

[31] Baumgartner, H. J., G. C. Hood, J. M. Monger, R. M. Roberts, and C. E. Sanborn. "Decomposition of concentrated hydrogen peroxide on silver I. Low temperature reaction and kinetics." Journal of Catalysis 2, no. 5 (1963): 405414. https://doi.org/10.1016/0021-9517(63)90105-2

[32] Othman, Norazila. "Design and development of 50-N Hydrogen Peroxide Monopropellant Rocket Thruster." PhD diss., Universiti Teknologi Malaysia, 2011.

[33] Kolaczkowski, S. T., R. Chao, S. Awdry, and A. Smith. "Application of a CFD code (FLUENT) to formulate models of catalytic gas phase reactions in porous catalyst pellets." Chemical Engineering Research and Design 85, no. 11 (2007): 1539-1552. https://doi.org/10.1205/cherd06226

[34] Ewis, Karem Mahmoud. "Analytical Solution of Modified Bingham Fluid Flow through Parallel Plates Channel Subjected to Forchheimer Medium and Hall Current Using Linearized Differential Transformation Method." Journal of Advanced Research in Numerical Heat Transfer 4, no. 1 (2021): 14-31.

[35] Kołodziej, Andrzej, Mieczysław Jaroszyński, Bożena Janus, Tadeusz Kleszcz, Joanna Łojewska, and Tomasz Łojewski. "An experimental study of the pressure drop in fluid flows through wire gauzes." Chemical Engineering Communications 196, no. 8 (2009): 932-949. https://doi.org/10.1080/00986440902743851

[36] Soares, Cíntia, Natan Padoin, Daliana Muller, Dachamir Hotza, and Carlos Renato Rambo. "Evaluation of resistances to fluid flow in fibrous ceramic medium." Applied Mathematical Modelling 39, no. 23-24 (2015): 7197-7210. https://doi.org/10.1016/j.apm.2015.02.014

[37] Zulkurnai, Fatin Farhanah, Wan Mohd Faizal Wan Mahmood, Norhidayah Mat Taib, and Mohd Radzi Abu Mansor. "Simulation of Combustion Process of Diesel and Ethanol Fuel in Reactivity Controlled Compression Ignition Engine." CFD Letters 13, no. 2 (2021): 1-11. https://doi.org/10.37934/cfdl.13.2.111

[38] Ansys. "ANSYS meshing user's guide." ANSYS 15317 (2013): 724-746.

[39] Vestnes, Frida. "A CFD-model of the Fluid Flow in a Hydrogen Peroxide Monopropellant Rocket Engine in ANSYS Fluent 16.2." Master's thesis, NTNU, 2016. 